\title{
Spectrum Sensing: To Cooperate or Not to Cooperate?
}

\author{
Dongliang Duan ${ }^{1}$, Liuqing Yang ${ }^{2 *}$, and Shuguang Cui ${ }^{3}$
}

1. Department of Electrical and Computer Engineering, University of Wyoming, Laramie, WY

2. Department of Electrical and Computer Engineering, Colorado State University, Fort Collins, CO

3. Department of Electrical and Computer Engineering, Texas A\&M University, College Station, TX

\section{Abstract}

While it is well accepted that cooperative spectrum sensing will significantly improve the sensing performance, the necessity of cooperation is not sufficiently appreciated. In this paper, by analyzing the spectrum sensing problem from the system perspective, we show that without cooperation, the performance will suffer from a fundamental tradeoff between reliability and efficiency. However, if cooperation is incorporated in the spectrum sensing process and the threshold is selected appropriately, the efficiencyreliability tradeoff in the non-cooperative case can be largely overcome by exploiting the cooperative diversity. These results show that cooperation in spectrum sensing is not just a luxury but a necessity.

Received on 10 November 2013; accepted on 08 December 2013; published on 11 April 2014

Keywords: Cognitive Radio, Cooperative Spectrum Sensing, Diversity, Efficiency and Reliability

Copyright (C) 2014 D. Duan et al., licensed to ICST. This is an open access article distributed under the terms of the Creative Commons Attribution license (http://creativecommons.org/licenses/by/3.0/), which permits unlimited use, distribution and reproduction in any medium so long as the original work is properly cited.

doi:10.4108/ws.1.1.e3

\section{Introduction}

In recent years, wireless services grow explosively both in variety and in quantity. While the current spectrum resources have already been assigned to various licensed services, the wireless industry faces the bottleneck of spectrum exhaustion. In the meantime, it is also reported that the current spectrum usage is highly inefficient [1] and many spectrum holes can be utilized at certain time or location. Under this condition, cognitive radio systems [2] provide an opportunity for unlicensed users (a.k.a. secondary users) to detect and initiate communications on these spectrum holes unused by licensed users (a.k.a. primary users) [3].

To achieve this objective, cognitive radio systems need to both maximally utilize opportunities of the spectrum holes and minimally interfere with the primary users. These are facilitated by the secondary user spectrum sensing capability to detect the spectrum holes, which corresponds to the "cognitive" part of the secondary system. Extensive research has already been conducted to improve the performance for both singleuser sensing and multi-user cooperative sensing (see e.g., [4-21]). However, most of these only treat spectrum

*Corresponding author. lqyang@engr.colostate.edu sensing as a detection problem without considering the context of the overall communications system.

In several recent papers, the interference from the secondary user system to the primary user system is evaluated (see e. g., [22-24]). In particular, [22] and [24] both report the observation that improved detection does not necessarily lead to reduced interference. Such inconsistency between detection and interference performance has motivated our work in this paper. In essence, the spectrum sensing schemes need to be designed in the context of the overall communications system, including both the primary and secondary ones.

Specifically, there are two error events in spectrum sensing, namely false alarm and missed detection. When false alarms occur, the secondary users will lose the opportunity of utilizing the spectrum, thus leading to reduced efficiency of spectrum utilization. When missed detections occur, the secondary users will initiate inappropriate communications over the spectrum in use and incur interference to the primary users, thus jeopardizing the reliability of the cognitive system. In this regard, we need to analyze spectrum sensing in terms of both the system efficiency and reliability.

It is usually believed that cooperation in spectrum sensing is just some luxury performance enhancement scheme over non-cooperative ones. However, our 
analyses and comparisons of non-cooperative sensing $(\mathrm{NCoS})$ vs. cooperative sensing $(\mathrm{CoS})$ with soft and hard information fusion will show that NCoS leads to a fundamental efficiency-reliability tradeoff, which can be largely overcome by CoS for any reasonable schemes. In essence, we claim that cooperation is actually a necessity rather than an option, in order to overcome the fundamental tradeoff.

The rest of the paper is organized as follows: the basic signal model and performance metrics under study are introduced in Section 2. The analysis of the fundamental efficiency-reliability tradeoff for noncooperative sensing is given in Section 3. Then, the necessity of cooperative spectrum sensing to overcome this fundamental tradeoff is demonstrated with numerical results for cooperative sensing with soft information fusion in Section 4 and for cooperative sensing with hard information fusion in Section 5, followed by concluding remarks in Section 6.

Notation: Subscripts ' $R$ ' and ' $E$ ' refer to reliabilityoriented and efficiency-oriented schemes, respectively; subscripts ' $S$ ' and ' $H$ ' refers to cooperative sensing with soft information fusion and hard information, respectively. $x \sim \mathcal{C N}\left(\mu, \sigma^{2}\right)$ denotes a circular symmetric complex Gaussian random variable $x$ with mean $\mu$ and variance $\sigma^{2} ; x \sim \operatorname{Ben}\left(p_{1}\right)$ denotes a random variable $x$ Bernoulli distributed with $\operatorname{Pr}(x=1)=p_{1} \cdot g(\gamma)=$ $o(f(\gamma))$ means $\lim _{\gamma \rightarrow+\infty} \frac{g(\gamma)}{f(\gamma)}=0 . \quad g(\gamma) \sim f(\gamma)$ means $\lim _{\gamma \rightarrow+\infty} \frac{g(\gamma)}{f(\gamma)}=c$ where $c$ is a non-zero constant with respect to $\gamma \cdot Q_{N}(x)=\int_{x}^{+\infty} \lambda^{N-1} \frac{e^{-\lambda}}{(N-1) !} d \lambda$ is the tailed cumulative distribution function of chi-square distribution with $2 \mathrm{~N}$ degrees of freedom.

\section{Problem Formulation}

\subsection{Signal Model}

In the spectrum sensing process, the sensing users will encounter the signals under the following two hypotheses:

$$
\begin{aligned}
& H_{0} \text { : absence of primary user } \\
& H_{1} \text { : presence of primary user. }
\end{aligned}
$$

As illustrated in $[5,14]$, after normalization by the noise power, the received signal is:

$$
\begin{aligned}
& r \mid H_{0}=n \sim \mathcal{C N}(0,1), \\
& r \mid H_{1}=h_{p, s} x+n \sim \mathcal{C N}(0, \gamma+1),
\end{aligned}
$$

where $x$ is the transmitted symbol of the primary user, $n$ is the normalized additive white Gaussian noise (AWGN), $h_{p, s}$ is the channel coefficient from the primary user to the secondary user and $\gamma=\frac{\sigma_{p, s}^{2} \mathcal{E}_{p}}{N_{0}}$ is the signalto-noise ratio (SNR) at the secondary user, where $N_{0}$ is the power of AWGN, $\mathcal{E}_{p}$ is the transmitted power of the primary user, and $\sigma_{p, s}^{2}$ is the variance or equivalently the average strength of channel $h_{p, s}$. Due to path loss, we know that $\gamma \sim \gamma_{1} d_{p, s}^{-K}$, where $d_{p, s}$ is the distance between the primary user and the sensing secondary user, $\gamma_{1}$ is the SNR when $d_{p, s}=1$, and $K$ is the path-loss exponent. In the remaining of this paper, a large SNR implies a small distance from the sensing secondary user to the primary user, and vice versa.

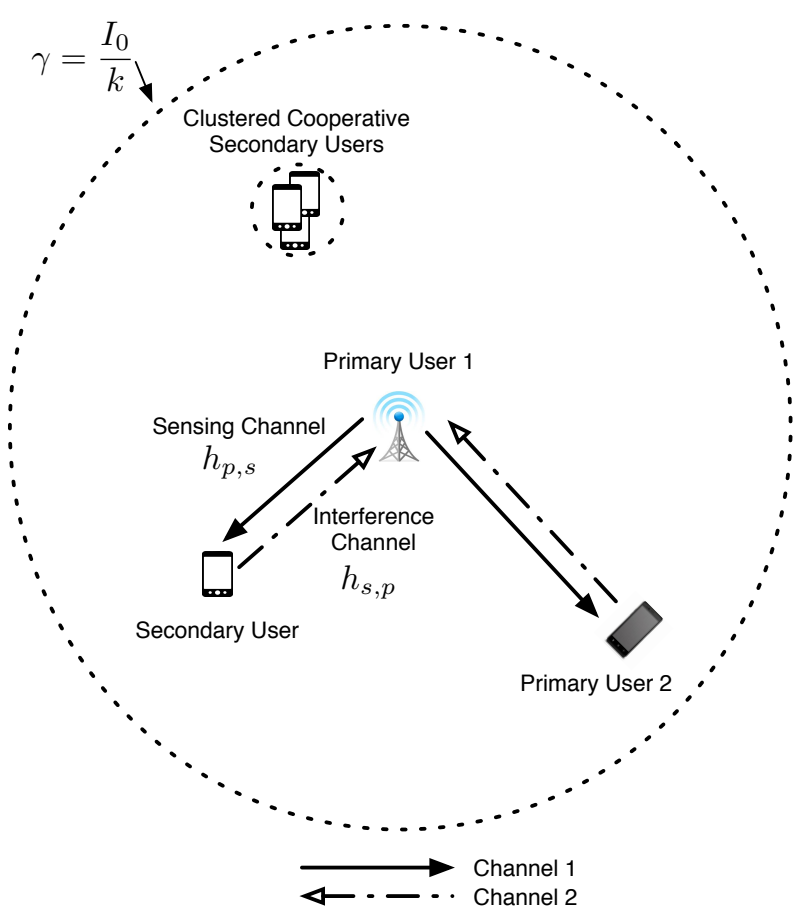

Figure 1. The system model.

In general cognitive radio systems, a secondary user senses the signal from a transmitter of the primary system and then incurs interference to the intended primary receiver, whose location can be quite unpredictable. This renders the interference analysis intractable and downgrades the usefulness of spectrum sensing, since the secondary user is not sensing the signal strength at the passive receiver side of the primary user system. To overcome this disagreement between sensing and interfering, and to simplify the analysis, [24] considered the setup where the primary network operating in the frequency division duplex mode as shown in Fig. $1^{1}$. In this case, the secondary user senses the downlink signals (channel 1 in the figure) and then transmits through the uplink channel (channel 2 in the figure), or vice versa. Accordingly, the location of the primary transmitter being sensed will also be the location of the receiver subject to the

\footnotetext{
${ }^{1}$ For simplicity, the communications among secondary users are not shown in the figure.
} 
interference from the secondary user (both at primary user 1 in Fig. 1). Hence the interference channel $h_{s, p}$ has the same average strength as the sensing channel $h_{p, s}$ since they undergo the same path-loss effect with $d_{p, s}=d_{s, p}$. That is, $\sigma_{s, p}^{2}=\sigma_{p, s}^{2}$, where $\sigma_{s, p}^{2}$ and $\sigma_{p, s}^{2}$ are the variances of $h_{s, p}$ and $h_{p, s}$, respectively.

\subsection{Performance Metrics}

In the context of the overall cognitive radio system, efficiency and reliability could be characterized by the capability of the secondary users in exploiting the unused spectrum and the average interference generated to disturb the active primary users. Hence, we will use the spectrum loss factor $\eta$ and the average interference $I$ as the performance metrics.

The spectrum loss factor is defined as the rate at which a false alarm occurs; thus

$$
\eta=P_{f} .
$$

For the average interference, one needs to consider both the occurrence rate and the average strength of the interference. The occurrence rate of interference is defined as the missed detection probability $P_{m d}$. The average interference strength depends on both the secondary user transmit power and the channel from the secondary user to the primary one. As a result, the average interference strength is $\mathcal{E}_{s} \sigma_{s, p}^{2}$, where $\mathcal{E}_{s}$ is the signal strength of the secondary users. With the signal model given in Section 2.1, this can be rewritten as $\mathcal{E}_{s} \sigma_{p, s}^{2}$. By the definition of $\gamma$, this is equal to $k \gamma$, where $k=\frac{\mathcal{E}_{s}}{\mathcal{E}_{p}} N_{0}$. It is reasonable to assume that the transmit power levels of the primary and secondary users are both kept constant for them to maintain a certain coverage range. Therefore, $k$ is a constant and the resultant average interference is given by

$$
I=k \gamma P_{m d} .
$$

From this expression, we notice that when a secondary user is far away from the primary user (low SNR), the detection performance must be bad; however, the average interference is not necessarily high due to the low interference strength. On the other hand, when a secondary user is close to the primary user (high SNR), the average interfering signal strength can be really high due to the high interference strength even though the detection performance may be better. In our performance analyses, we will focus on the high SNR range, and the performance at low SNR will be shown numerically.

With these performance metrics, one can have two spectrum sensing strategies emphasizing either the system reliability or the system efficiency. For reliability-oriented systems, the average interference $I$ is minimized under the constraint of a preset spectrum loss factor $\eta$; while for efficiency-oriented systems, the spectrum loss factor $\eta$ is minimized under the constraint of a tolerable average interference $I$.

\section{Analysis of Non-Cooperative Sensing}

For NCoS, the optimal detector is the energy detector under our setup [25]:

$$
\lambda=|r|^{2} \underset{H_{0}}{\stackrel{H_{1}}{\gtrless}} \theta,
$$

where $\theta$ is the decision threshold. Accordingly, the false alarm and missed detection probabilities are given as

$$
P_{f}=e^{-\theta},
$$

and

$$
P_{m d}=1-e^{-\frac{\theta}{\gamma+1}}
$$

respectively.

\subsection{Reliability-Oriented Scheme with $\eta \leq \eta_{0}$}

Under this scheme, the threshold should be chosen to minimize the average interference $I$ while ensuring that the efficiency is maintained at a preset level $P_{f}=\eta_{0}$. From Eq. (5) we obtain

$$
\theta_{R}=-\ln \eta_{0}
$$

Thus, the average interference to the primary user due to inappropriate secondary communications is

$$
\begin{aligned}
I_{R} & =k \gamma P_{m d} \\
& =k \gamma\left(1-\left(\eta_{0}\right)^{\frac{1}{\gamma+1}}\right) .
\end{aligned}
$$

As $\gamma \rightarrow+\infty, \frac{1}{\gamma+1} \rightarrow 0$. Taking Taylor series expansion of $\eta_{0}^{\frac{1}{\gamma+1}}$ with respect to $\frac{1}{\gamma+1}$ around 0 , we have

$$
\begin{aligned}
I_{R} & =k \gamma\left(1-\left(1+\frac{1}{\gamma+1} \ln \eta_{0}+o\left(\frac{1}{1+\gamma}\right)\right)\right) \\
& =-k \ln \eta_{0} \frac{\gamma}{\gamma+1}+k \gamma o\left(\frac{1}{1+\gamma}\right) \\
& \approx k \ln \frac{1}{\eta_{0}} .
\end{aligned}
$$

That is, with the reliability-oriented scheme, the average interference $I_{R}$ approaches constant $k \ln \eta_{0}$ as $\gamma$ increases. The numerical plot is shown in Fig. 2.

\subsection{Efficiency-Oriented Scheme with $I \leq I_{0}$}

Similarly, in order to minimize the spectrum loss factor $\eta$ under the interference constraint, the threshold should be selected such that $P_{m d}=\min \left(\frac{I_{0}}{k \gamma}, 1\right)$. Notice 


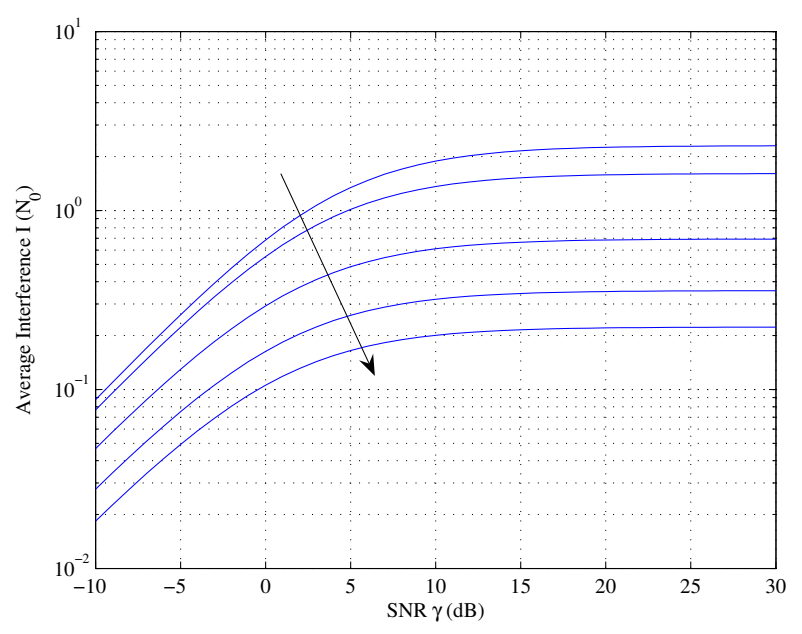

Figure 2. The reliability-oriented scheme for NCoS. $k=N_{0}$. Along the direction of the arrow, $\eta_{0}=0.1,0.2,0.5,0.7,0.8$.

that when $\gamma<\frac{I_{0}}{k}$ (out of the circle in Fig. 1), the spectrum loss factor can approach zero since the average interference constraint is satisfied even when $P_{m d}=1$. In other words, it does not matter whether or not the presence of the primary user is detected, simply because the interference induced by the secondary users is negligible.

For $\gamma \geq \frac{I_{0}}{k}$, setting $P_{m d}=\frac{I_{0}}{k \gamma}$ together with Eq. (6), we obtain

$$
\theta_{E}=-(\gamma+1) \ln \left(1-\frac{I_{0}}{k \gamma}\right)
$$

With this threshold, the spectrum loss factor is

$$
\eta_{E}=e^{(\gamma+1) \ln \left(1-\frac{I_{0}}{k \gamma}\right)} .
$$

As $\gamma \rightarrow+\infty$, by Taylor series expansion, we have

$$
\ln \left(1-\frac{I_{0}}{k \gamma}\right)=-\frac{I_{0}}{k \gamma}+o\left(\frac{1}{\gamma}\right) .
$$

Hence we obtain

$$
\begin{aligned}
\eta_{E} & =e^{-\frac{I_{0}}{k} \frac{\gamma+1}{\gamma}+(\gamma+1) o\left(\frac{1}{\gamma}\right)} \\
& \approx e^{-\frac{I_{0}}{k}}
\end{aligned}
$$

This means that with the efficiency-oriented scheme, the system performance also saturates at high SNR. The numerical plot is shown in Fig. $3^{2}$.

From the analyses above, we see that for NCoS, the reliability and efficiency exhibit a persistent tradeoff: when one is lower, the other is inevitably higher. The saturating effects in Figs. 2 and 3 confirm that such tradeoff cannot be improved by the SNR increase.

\footnotetext{
$\overline{{ }^{2} \text { Without loss of generality, we set } k=N_{0} \text { in all our numerical studies. }}$
}

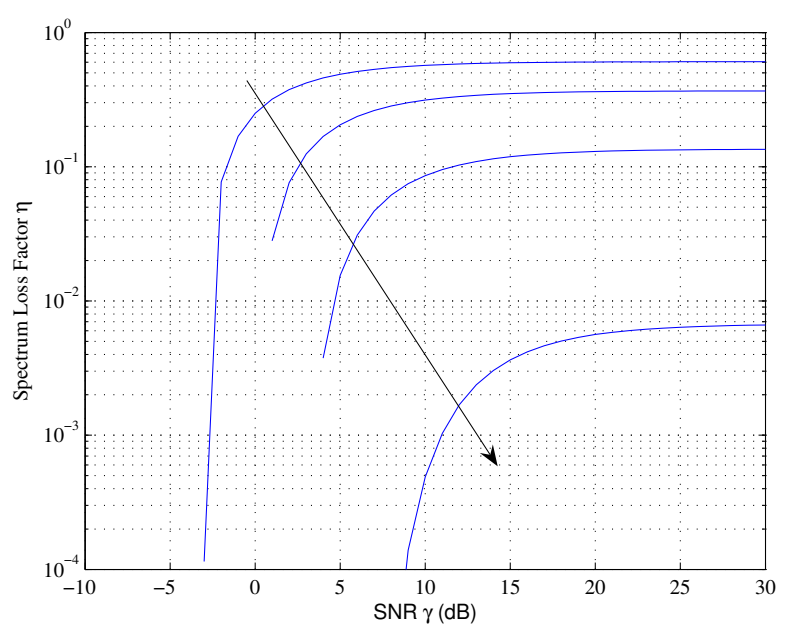

Figure 3. The efficiency-oriented scheme for NCoS. $k=N_{0}$. Along the direction of the arrow, $I_{0}=0.5 N_{0}, N_{0}, 2 N_{0}, 5 N_{0}$.

\section{Analysis of Cooperative Sensing with Soft Information Fusion (SCoS)}

With cooperative sensing, a fusion center is collecting information from geographically distributed local secondary sensing users to make a global decision. If the channels from the local secondary users to the fusion center have sufficiently high bandwidth, we can assume that the fusion center can receive roughly exact sensed signal values $r_{1}, r_{2}, \ldots, r_{N}$ from different sensing users, where $N$ is the total number of sensing users. For practical considerations, we study the case where the $N$ secondary users are geographically clustered to cooperate. This is based on the consideration that the cooperative detection of the primary user presence is only meaningful when the cooperating secondary users are subject to the same primary user activity and are thus posing similar interferences to the primary receiver. As a result, the cooperating users share the same level of large-scale path loss as shown in Fig. 1. In this case, the SNRs in their received signals are the same and $r_{i}$ 's are independent identically distributed (i.i.d). In this case, the optimal detector is again an energy detector [25]:

$$
\lambda_{S}=\sum_{i=1}^{N}\left|r_{i}\right|^{2} \underset{H_{0}}{\stackrel{H_{1}}{\gtrless}} \theta_{S},
$$

where $\theta_{S}$ is the decision threshold at the fusion center.

Since $r_{i}$ 's are independent circular symmetric complex Gaussian variables, the false alarm and missed detection probabilities are respectively

$$
P_{f, S}(N)=Q_{N}\left(\theta_{S}\right)
$$

and

$$
P_{m d, S}(N)=1-Q_{N}\left(\frac{\theta_{S}}{\gamma+1}\right) .
$$




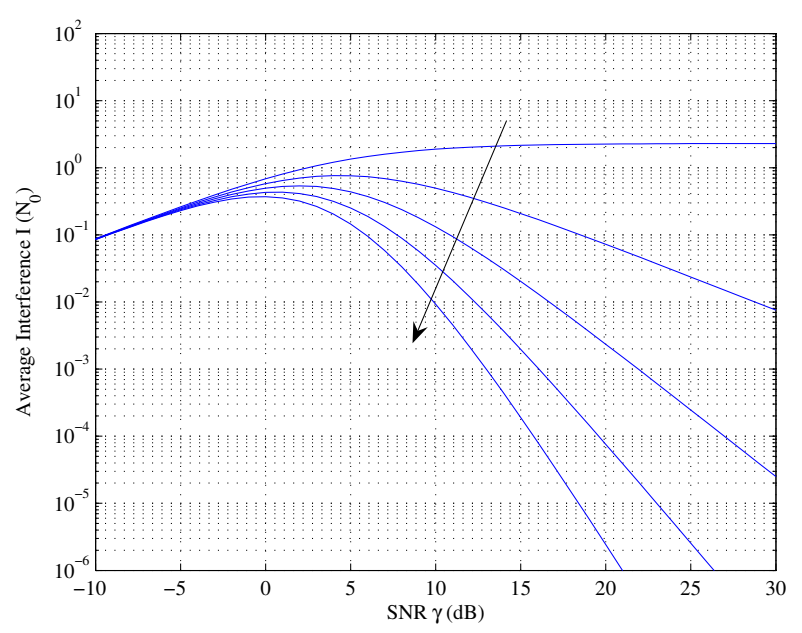

Figure 4. The reliability-oriented scheme for SCoS with $\eta \leq$ 0.01. $k=N_{0}$. Along the direction of the arrow, $N=1,2,3,4,5$.

\subsection{Reliability-Oriented Scheme with $\eta \leq \eta_{0}$}

Similar to the case of NCoS, in the reliability-oriented scheme, we set $P_{f, S}=\eta_{0}$ and obtain the threshold according to Eq. (15) as:

$$
\theta_{R, S}(N)=Q_{N}^{-1}\left(\eta_{0}\right) .
$$

With this threshold, we have

$$
I_{R, S}(N)=k \gamma \int_{0}^{\frac{Q_{N}^{-1}\left(\eta_{0}\right)}{\gamma+1}} \lambda^{N-1} \frac{e^{-\lambda}}{(N-1) !} d \lambda .
$$

As $\gamma \rightarrow+\infty, \frac{Q_{N}^{-1}\left(\eta_{0}\right)}{\gamma+1} \rightarrow 0$. Thus, within the integration interval $\lambda \in\left[0, \frac{Q_{N}^{-1}\left(\eta_{0}\right)}{\gamma+1}\right]$, we have $e^{-\lambda} \approx 1$ for large $\gamma$. Thus,

$$
\begin{aligned}
I_{R, S}(N) & \approx k \gamma \int_{0}^{\frac{Q_{N}^{-1}\left(\eta_{0}\right)}{\gamma+1}} \frac{\lambda^{N-1}}{(N-1) !} d \lambda \\
& =\left.k \gamma \cdot \frac{\lambda^{N}}{N !}\right|_{0} ^{\frac{Q_{N}^{-1}\left(\eta_{0}\right)}{\gamma+1}} \\
& =k \gamma \frac{\left(Q_{N}^{-1}\left(\eta_{0}\right)\right)^{N}}{N !(\gamma+1)^{N}} \\
& \sim \gamma^{-(N-1)} .
\end{aligned}
$$

This result indicates that system reliability has a diversity order of $(N-1)$. The numerical plot is given in Fig. 4.

\subsection{Efficiency-Oriented Scheme with $I \leq I_{0}$}

Similar to the NCoS case, in the efficiency-oriented scheme, we can set $P_{m d, S}(N)=\min \left(\frac{I_{0}}{k \gamma}, 1\right)$, which means that $P_{m d, S}(N)=1$ for $\gamma<\frac{I_{0}}{k}$.
When $\gamma \geq \frac{I_{0}}{k}$, setting $P_{m d, S}(N)=\frac{I_{0}}{k \gamma}$ together with Eq. (16), we have the decision threshold as

$$
\theta_{E, S}(N)=(\gamma+1) Q_{N}^{-1}\left(1-\frac{I_{0}}{k \gamma}\right)
$$

When $\gamma \rightarrow+\infty, P_{m d, S}(N)=\frac{I}{k \gamma} \rightarrow 0$; then the integral upper bound $\frac{\theta_{E, S}(N)}{\gamma+1} \rightarrow 0$. Hence, within the integration interval $\left[0, \frac{\theta_{E, N}}{\gamma+1}\right]$, similar approximation as in Section 5.1 can be utilized. As a result:

$$
\frac{I}{k \gamma}=P_{m d, N} \approx \frac{\left(\theta_{E, S}(N)\right)^{N}}{N !(\gamma+1)^{N}} .
$$

That is,

$$
\theta_{E, S}(N) \approx(\gamma+1)\left(\frac{N ! I}{k \gamma}\right)^{\frac{1}{N}} \sim \gamma^{1-\frac{1}{N}} .
$$

With threshold $\theta_{E, S}(N)$, the spectrum loss factor is

$$
\begin{aligned}
\eta_{E, S}(N) & =P_{f, S}(N)=\int_{\theta_{E, S}(N)}^{+\infty} \lambda^{N-1} \frac{e^{-\lambda}}{(N-1) !} d \lambda \\
& =\left(\sum_{i=0}^{N-1} \frac{\left(\theta_{E, S}(N)\right)^{i}}{i !}\right) e^{-\theta_{E, S}(N)} .
\end{aligned}
$$

Recall that we have shown that $\theta_{E, S}(N)$ increases as $\gamma$ increases. Hence as $\gamma \rightarrow+\infty$, the summation in $\eta_{E, S}(N)$ will be dominated by the term with the highest order. Together with the expression of $\theta_{E, S}(N)$ in Eq. (19), we obtain

$$
\begin{aligned}
\eta_{E, S}(N) & \approx \frac{\left(\theta_{E, S}(N)\right)^{N-1}}{(N-1) !} e^{-\theta_{E, S}(N)} \\
& \sim \gamma^{\left((N-1)\left(1-\frac{1}{N}\right)\right)} e^{-\gamma^{\left(1-\frac{1}{N}\right)}} \\
& =o\left(\gamma^{-p}\right),
\end{aligned}
$$

where $p$ is an arbitrary positive real number. This implies that the system efficiency has a diversity order of infinity. The numerical plot is given in Fig. 5.

In this section, we see that thanks to cooperative sensing with soft information fusion, for both efficiencyand reliability-oriented schemes, these figures of merit can be consistently improved as SNR increases. This is in sharp contrast to the persistent tradeoff that we observed in the non-cooperative case.

\section{Analysis of Cooperative Sensing with Hard Information Fusion (HCoS)}

In order for the soft-information based sensing scheme described in Section 4 to work, the bandwidth of the channel between the sensing secondary users and the fusion center has to be very large. This usually requires a backbone wired communication system to 


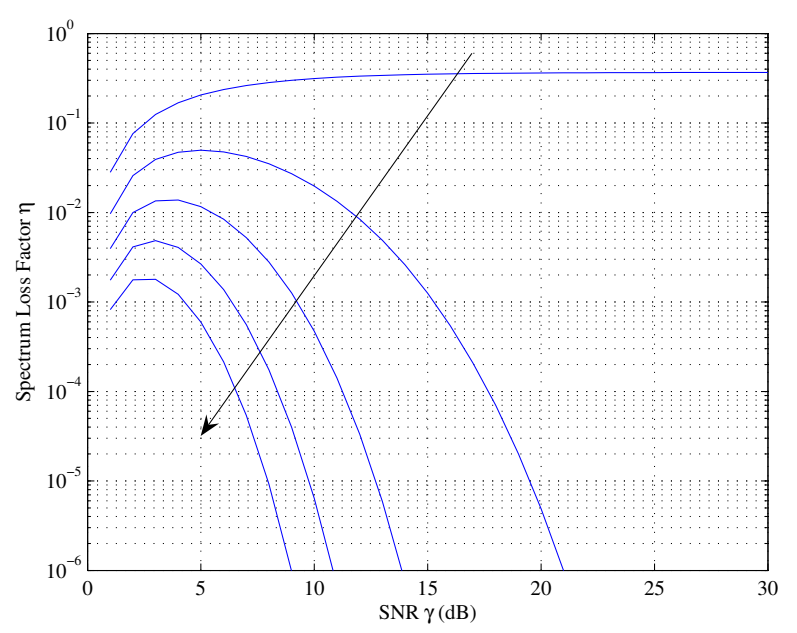

Figure 5. The efficiency-oriented scheme for SCoS with $I \leq N_{0}$. $k=N_{0}$. Along the direction of the arrow, $N=1,2,3,4,5$.

connect the fusion center and the secondary users, which is impractical for mobile secondary devices. Hence, usually quantizations are applied to the received signal at the local secondary user and only a limited number of bits will be sent to the fusion center for a global decision. Although multi-bit quantization can be made (see e.g. $[13,18]$ ), in this paper, we only study the extreme case where the local quantizations are simply binary.

In this case, each sensing secondary user makes a local one-bit decision $d_{i}$ according to the optimal rule in the NCoS case,

$$
|r|^{2} \underset{d_{i}=0}{d_{i}=1} \theta_{l},
$$

where $\theta_{l}$ is the local decision threshold adopted by all secondary sensing users. Hence, at the fusion center, the received local decisions follow an i.i.d. Bernoulli distribution with parameters $P_{f, l}$ and $1-P_{m d, l}$ under $H_{0}$ and $H_{1}$, respectively:

$$
\begin{aligned}
& d_{i} \mid H_{0} \sim \operatorname{Ber}\left(P_{f, l}\right), \\
& d_{i} \mid H_{1} \sim \operatorname{Ber}\left(1-P_{m d, l}\right),
\end{aligned}
$$

where

$$
P_{f, l}=e^{-\theta_{l}}
$$

is the local false alarm probability, and

$$
P_{m d, l}=1-e^{-\frac{\theta_{l}}{\gamma+1}}
$$

is the local missed detection probability. Then, the decision rule at the fusion center is cast as

$$
\lambda_{H}=\sum_{i=1}^{N} d_{i} \underset{H_{0}}{\stackrel{H_{1}}{\gtrless}} \theta_{H},
$$

where $\lambda_{H}$ and $\theta_{H}$ are the decision statistics and the decision threshold at the fusion center, respectively. Naturally, the HCoS involves two thresholds, namely the local threshold $\theta_{l}$ and the fusion threshold $\theta_{H}$ in the performance optimization process. However, in our previous work [20], we have shown that as $\gamma \rightarrow$ $+\infty$, the optimal fusion rule is the 'OR' rule, i.e., the fusion center will declare the absence of the primary user only when all local sensing secondary users make universal decisions $d_{i}=0$ and will declare the presence of primary user as long as at least one of the local sensing secondary users make decision $d_{i}=1$. Hence, the optimal fusion threshold is set as $\theta_{H}^{o}=1$.

Under the 'OR' rule, the global false alarm and missed detection probabilities depend on the local threshold $\theta_{l}$ as

$$
\begin{aligned}
P_{f, H}(N) & =1-\left(1-P_{f, l}\right)^{N} \\
& =1-\left(1-e^{-\theta_{l}}\right)^{N},
\end{aligned}
$$

and

$$
\begin{aligned}
P_{m d, H}(N) & =\left(P_{m d, l}\right)^{N} \\
& =\left(1-e^{-\frac{\theta_{l}}{\gamma+1}}\right)^{N} .
\end{aligned}
$$

\subsection{Reliability-Oriented Scheme with $\eta \leq \eta_{0}$}

Similar to the cases of NCoS and SCoS, in the reliabilityoriented scheme, we set $P_{f, H}=\eta_{0}$ and obtain the local threshold according to Eqs. (22) and (25):

$$
\theta_{R, l}(N)=-\ln \left(1-\left(1-\eta_{0}\right)^{\frac{1}{N}}\right) .
$$

With this local threshold and by Eq. (26), we have

$$
\begin{aligned}
I_{R, H}(N) & =k \gamma P_{m d, H}(N) \\
& =k \gamma\left(1-e^{-\frac{\theta_{R, l}(N)}{\gamma+1}}\right)^{N} \\
& =k \gamma\left(-\frac{1}{\gamma+1} \ln \left(\theta_{R, l}(N)\right)+o\left(\frac{1}{\gamma+1}\right)\right)^{N} \\
& \approx k \gamma\left(\frac{1}{\gamma+1}\right)^{N}\left(-\ln \left(-\ln \left(1-\left(1-\eta_{0}\right)^{\frac{1}{N}}\right)\right)\right)^{N} \\
& \sim \gamma^{-(N-1)},
\end{aligned}
$$

where Taylor series expansion is applied as in Eq. (9) for $\gamma \rightarrow+\infty$.

This result indicates that the system reliability for HCoS exhibits the same diversity order of $(N-1)$ as for SCoS. The numerical plot is given in Fig. 6.

\subsection{Efficiency-Oriented Scheme with $I \leq I_{0}$}

Similar to NCoS and SCoS cases, in the efficiencyoriented scheme, we set $P_{m d, H}(N)=\min \left(\frac{I_{0}}{k \gamma}, 1\right)$, which similarly means that $P_{m d, H}(N)=1$ for $\gamma<\frac{I_{0}}{k}$. 


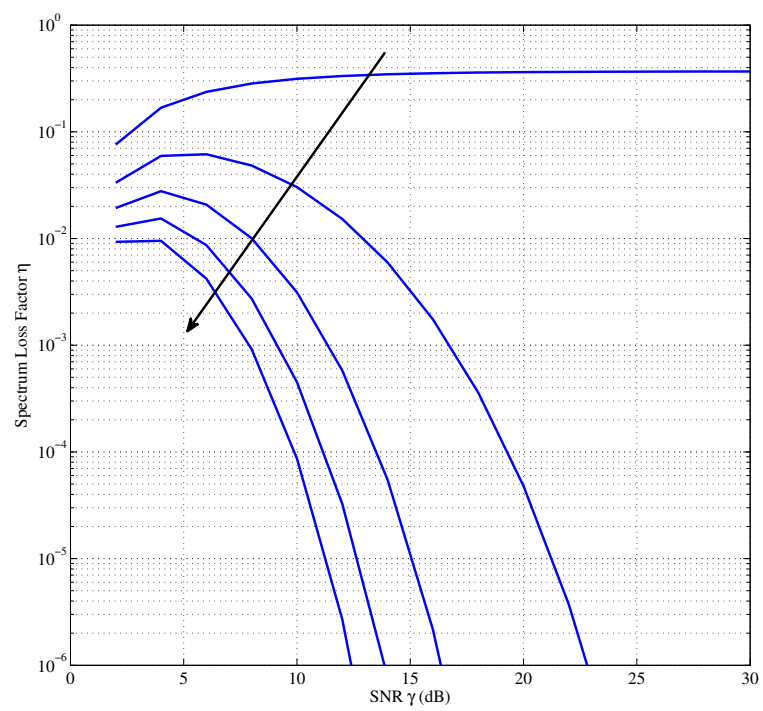

Figure 6. The reliability-oriented scheme for $\mathrm{HCOS}$ with $\eta \leq$ 0.01. $k=N_{0}$. Along the direction of the arrow, $N=1,2,3,4,5$.

When $\gamma \geq \frac{I_{0}}{k}$, by setting $P_{m d, H}(N)=\frac{I_{0}}{k \gamma}$, together with Eqs. (23) and (26), the local decision threshold is obtained as

$$
\theta_{E, l}(N)=-(\gamma+1) \ln \left(1-\left(\frac{I_{0}}{k \gamma}\right)^{\frac{1}{N}}\right)
$$

Then, according to Eqs. (22) and (25), as $\gamma \rightarrow+\infty$, the spectrum loss factor is

$$
\begin{aligned}
\eta_{E, H}(N) & =P_{f, H}(N)=1-\left(1-e^{-\theta_{E, l}(N)}\right)^{N} \\
& \approx N e^{-\theta_{E, l}(N)} \\
& =N\left(\frac{1}{1-\left(\frac{I_{0}}{k \gamma}\right)^{\frac{1}{N}}}\right)^{\gamma+1} \\
& \sim o\left(\gamma^{-p}\right),
\end{aligned}
$$

where $p$ is an arbitrary positive real number. Therefore, similar to SCoS, the system efficiency for HCoS also has a diversity order of infinity. The numerical plot is given in Fig. 7.

In this section, we see that although local binary quantizations are made at local sensing secondary users, cooperative sensing with hard information fusion can also greatly improve the figures of merit for both efficiency- and reliability-oriented schemes, similar as SCoS. This implies that even a very simple cooperative scheme can help overcome the fundamental efficiencyreliability tradeoff incurred by NCoS.

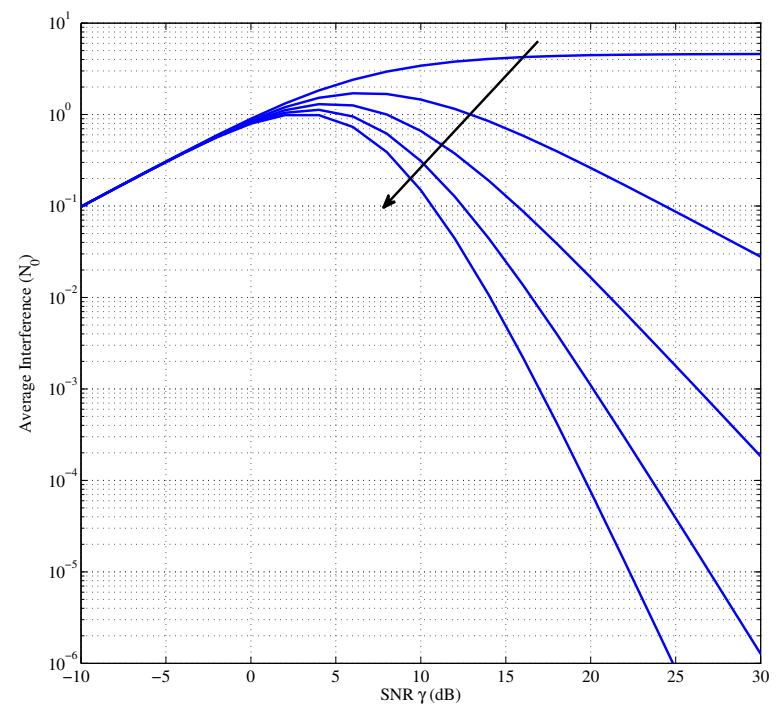

Figure 7. The efficiency-oriented scheme for $\mathrm{HC} 0 \mathrm{~S}$ with $I \leq N_{0}$. $k=N_{0}$. Along the direction of the arrow, $N=1,2,3,4,5$.

\section{Conclusions}

In this paper, we analyzed the spectrum sensing performance of non-cooperative sensing (NCoS) and cooperative sensing with soft $(\mathrm{SCoS})$ and hard information fusion $(\mathrm{HCoS})$ in the context of a cognitive radio system, and gained some insightful observations on the role of cooperation. To capture the system tradeoff between efficiency and reliability, we introduced the spectrum loss factor $\eta$ and the average interference $I$ as the performance metrics. With these metrics, we analyzed both reliability-oriented and efficiency-oriented schemes for NCoS, SCoS, and HCoS. Results show that the seemingly unavoidable efficiency-reliability tradeoff in NCoS can be largely avoided by exploiting diversity via cooperating sensing. This is the case not only with SCoS collecting perfect information from local sensors but also with $\mathrm{HCoS}$ collecting only quantized information. In a nutshell, cooperation in spectrum sensing is not just a luxury but a necessity.

Acknowledgement. This paper was in part supported by National Science Foundation under awards CNS-1265227, CNS-1343155, ECCS-1305979 and ECCS-1232305.

\section{References}

[1] Наyкin, S. (2005) Cognitive radio: brain-empowered wireless communications. IEEE Journal on Selected Areas in Communications 23(2): 201-220.

[2] Mitola III, J. and Maguire JR., G. (1999) Cognitive radio: making software radios more personal. IEEE Personal Communications 6(4): 13-18.

[3] Staple, G. and Werbach, K. (2004) The end of spectrum scarcity. IEEE Spectrum 41(3): 48-52. 
[4] Cabric, D., Tkachenko, A. and Brodersen, R.W. (2006) Experimental study of spectrum sensing based on energy detection and network cooperation. In Proc. ACM 1st Int. Workshop on Technology and Policy for Accessing Spectrum (TAPAS) (Boston, MA): 1-8.

[5] Hong, S., Vu, M.H. and Tarokh, V. (2008) Cognitive sensing based on side information. In IEEE Sarnoff Symposium (Princeton, NJ): 1-6.

[6] Quan, Z., Cui, S. and SAyed, A.H. (2008) Optimal linear cooperation for spectrum sensing in cognitive radio networks. IEEE Journal of Selected Topics in Singal Processing 2(1): 28-40.

[7] Zhang, W. and Letaief, K. (2008) Cooperative spectrum sensing with transmit and relay diversity in cognitive radio networks. IEEE Trans. on Wireless Communications 7(12): 4761-4766.

[8] Quan, Z., Cui, S., Poor, H. and Sayed, A. (2008) Collaborative wideband sensing for cognitive radios. IEEE Signal Processing Magazine 25(6): 60-73.

[9] Unnikrishnan, J. and Veeravalli, V.V. (2008) Cooperative sensing for primary detection in cognitive radio. IEEE Journal of Selected Topics in Singal Processing 2(1): $18-27$.

[10] Wang, H., hui YANG, E., Zhao, Z. and Zhang, W. (2009) Spectrum sensing in cognitive radio using goodness of fit testing. IEEE Trans. on Wireless Communications 8(11): 5427-5430.

[11] Quan, Z., Cui, S., Sayed, A. and Poor, H. (2009) Optimal multiband joint detection for spectrum sensing in cognitive radio networks. IEEE Trans. on Signal Processing 57(3): 1128-1140.

[12] Zhang, W., Mallik, R. and Letaief, K. (2009) Optimization of cooperative spectrum sensing with energy detection in cognitive radio networks. IEEE Trans. on Wireless Communications 8(12): 5761-5766.

[13] Chaudhari, S., Lunden, J., Koivunen, V. and Poor, H.V. (2010) Cooperative sensing with imperfect reporting channels: Hard decisions or soft decisions? IEEE Trans. on Signal Processing 60(1): 18-28.

[14] Duan, D., Yang, L. and Principe, J. (2010) Cooperative diversity of spectrum sensing for cognitive radio systems. IEEE Trans. on Signal Processing 58(6): 32183227.

[15] Peh, E., Liang, Y.C., Guan, Y.L. and Zeng, Y. (2010) Cooperative spectrum sensing in cognitive radio networks with weighted decision fusion schemes. IEEE Trans. on Wireless Communications 9(12): 3838-3847.

[16] Kim, S.J., Dall'Anese, E. and Giannakis, G.B. (2011) Cooperative spectrum sensing for cognitive radios using kriged kalman filtering. IEEE Journal of Selected Topics in Signal Processing 5(1): 24-36.

[17] Shen, L., WANG, H., Zhang, W. and Zhao, Z. (2011) Blind spectrum sensing for cognitive radio channels with noise uncertainty. IEEE Trans. on Wireless Communications 10(6): 1721-1724.

[18] DuAn, D. and YANG, L. (2012) Cooperative spectrum sensing with ternary local decisions. IEEE Communications Letters 16(9): 1512-1515.

[19] Duan, D., Yang, L. and Scharf, L.L. (2012) Optimal detection fusion by large deviation analysis. In Proceedings of European Signal Processing Conference (EUSIPCO) (Bucharest, Romania): 744-748.

[20] Duan, D., Yang, L. and Scharf, L.L. (2012) The optimal fusion rule for cooperative spectrum sensing from a diversity perspective. In Proc. of Asilomar Conf. on Signals, Systems, and Computers (Pacific Grove, CA): 1056-1060.

[21] Yucek, T. and Arslan, H. (2009) A survey of spectrum sensing algorithms for cognitive radio applications. IEEE Communications Surveys Tutorials 11(1): 116-130.

[22] Duan, D., Yang, L. and Principe, J. (2009) Detectioninterference dilemma for spectrum sensing in cognitive radio systems. In Proc. of MILCOM Conf. (Boston, MA): $1-7$.

[23] Liang, Y.C., Zheng, Y., Peh, E.C.Y. and Hoang, A.T. (2008) Sensing-throughtput tradeoff for cognitive radio networks. IEEE Trans. on Wireless Communications 7(4): 1326-1337.

[24] Rabbachin, A., QueK, T.Q.S. and Win, M.Z. (2011) Cognitive network interference. IEEE Journal on Selected Areas in Communications 29(2): 480-493.

[25] Poor, H.V. (1994) An Introduction to Signal Detection and Estimation (Springer-Verlag), 2nd ed. 